\title{
Atención visual hacia el etiquetado de bebidas alcohólicas: un estudio exploratorio basado en eye-tracking
}

\section{Visual attention to alcohol labels: an exploratory eye-tracking experiment}

\author{
Carlos Sillero-Rejon*,**,***, Olivia Maynard*******, José-Angel Ibáñez-Zapata***. \\ * Centro de Estudios sobre Tabaco y Alcohol del Reino Unido, Escuela de Ciencias Psicológicas. Universidad de Bristol. \\ Reino Unido. ** Unidad de Epidemiología Integrativa (IEU) MRC. Universidad de Bristol. Reino Unido. *** Departamento \\ de Marketing e Investigación de Mercados. Universidad de Granada. España.
}

\section{Resumen}

El actual etiquetado de bebidas alcohólicas pasa desapercibido por los consumidores. Además, la legislación europea exime a la industria de bebidas alcohólicas de incluir advertencias sanitarias en sus envases. Este estudio pretende explorar cómo el tamaño, el diseño y el contenido alcohólico de las bebidas influyen en la atención visual prestada al etiquetado de la graduación alcohólica; y cómo el tamaño y el contenido alcohólico influyen en la atención visual prestada hacia una advertencia sanitaria. Usando un diseño experimental, se monitorizaron los movimientos oculares de 64 participantes mientras visualizaban envases de cerveza con diferente graduación $(0,4 \%, 4,6 \%$ vs. $15 \%)$. Se midió el número de fijaciones hacia el etiquetado de la graduación alcohólica, manipulando su tamaño y el diseño como factores inter-sujetos. En los envases presentados a la mitad de los participantes se incluyó una advertencia sanitaria, manipulando su tamaño como factor inter-sujeto. Los resultados muestran claras evidencias estadísticas de que el número de fijaciones es superior cuando la graduación alcohólica se muestra con un mayor tamaño y mediante un semáforo nutricional. Asimismo, los resultados relevan un mayor número de fijaciones hacia la advertencia sanitaria cuando esta tiene un tamaño mayor y obtiene diferentes niveles de atención visual dependiendo de la graduación alcohólica del envase. En conclusión, el estudio pone de manifiesto que el actual etiquetado de los envases de bebidas alcohólicas es insuficiente para captar la atención de los consumidores y sugiere que, si se persigue lograr un incremento de la atención prestada, el etiquetado debe mostrarse con un mayor tamaño y con un diseño gráfico. Palabras clave: Alcohol; Etiquetado; Advertencia; Atención; Eye-tracking.

\begin{abstract}
Current alcohol labelling goes unnoticed by consumers. In addition, EU legislation does not obligate the alcohol industry to include any health warning labels on alcohol packagings. This study aims to explore how the size and design of alcohol by volume (ABV) labels, along with the alcohol strength presented on these labels, influence visual attention toward them. We also examine how label size and alcohol strength influence visual attention toward a health warning label on alcoholic beverages. Using an experimental human laboratory design, we tracked the eye-movements of 64 participants while they viewed beers with different $\mathrm{ABV}(0.4 \%, 4.6 \%$ vs. $15 \%)$. We measured the number of fixations toward ABV labelling which varied in size and design (text-only vs. traffic light). A health warning label was also included on the beers for half of the participants and size was manipulated as a between-subject factor. Results showed strong evidence that the number of fixations was higher when the $\mathrm{ABV}$ labels were larger and used a traffic light system. Likewise, we found a higher number of fixations toward larger health warning labels and differences in visual attention depending on the $A B V$ content presented. In conclusion, this study indicates that current alcohol labelling is insufficient to draw the attention of consumers and suggests that future alcohol labelling must be larger and with a graphic design to attract attention.

Key Words: Alcohol; Labelling; Health warning; Attention; Eye-tracking.
\end{abstract}


$\mathrm{E}$ 1 alcohol es la tercera causa de morbilidad y mortalidad en la Unión Europea (UE), y Europa es la región del mundo con el mayor consumo de alcohol (Anderson, Moller, Galea, y World Health Organization, 2012). A pesar de ello, la legislación de la UE solo requiere la inclusión de etiquetas que muestren el volumen de alcohol y no obliga a insertar advertencias sanitarias en los envases de bebidas alcohólicas (Farke, 2011). En España, lugar donde se llevó a cabo este estudio, el volumen de alcohol suele mostrase de forma pequeña, mediante solo texto y suele encontrarse en el dorso del producto; cumpliendo, así, con los requisitos mínimos impuestos por la legislación actual (Parlamento Europeo y del Consejo, 2011); mientras que las advertencias sanitarias son implementadas de forma voluntaria (Farke, 2011). Por tanto, este etiquetado no suele ser identificado con facilidad, ya que suele ocupar poco espacio y rara vez incluye componentes gráficos o es actualizado con periodicidad (Wilkinson y Room, 2009). En otros países europeos, como el Reino Unido, las empresas productoras de alcohol se comprometieron a etiquetar sus bebidas alcohólicas con información para un consumo responsable. No obstante, los estudios muestran que los consumidores prestan poca atención a este etiquetado voluntario (Kersbergen y Field, 2017). La literatura sugiere que un mayor tamaño de estas etiquetas tendría el potencial de atraer mayores niveles de atención (Farke, 2011; Kersbergen y Field, 2017; Truitt et al., 2002). Aunado a ello, un aumento de su visibilidad mediante diseños gráficos, lo cual es infrecuente en Europa (Wilkinson et al., 2009), podría atraer y mantener la atención, como se ha demostrado en las investigaciones sobre el etiquetado en la industria alimentaria (Graham, Orquin y Visschers, 2012). Por otra parte, se ha sugerido incluir advertencias sanitarias en los envases de bebidas alcohólicas similares a las que ya se usan en los envases de tabaco (Thomson, Vandenberg y Fitzgerald, 2012), dada su efectividad a la hora de cambiar el comportamiento (Hammond, 2011) y al comunicar los riesgos adheridos al consumo de tabaco (Noar et al., 2015). Recientemente, Blackwell, Drax, Attwood, Munafo y Maynard (2018) concluyeron que se podría optimizar las etiquetas que muestran las unidades del alcohol para una mejor comprensión del contenido de alcohol y, además, sugirieron que se debería incluir una advertencia sanitaria junto a este etiquetado para una mayor comprensión de los riesgos que conlleva el consumo alcohol y para disuadir conductas asociadas a un consumo de riesgo.

El presente estudio pretende explorar el nivel de atención visual hacia una serie de diferentes etiquetas del volumen de alcohol y hacia una advertencia sanitaria en los envases de bebidas alcohólicas. Como principal hipótesis se plantea que unas etiquetas más grandes (tanto para el volumen de alcohol como para la advertencia sanitaria) y la inclusión de un componente gráfico, como un semá- foro nutricional (para el volumen de alcohol) frente a la información mostrada mediante solo texto, aumentarán los niveles de atención visual. También se considera que el nivel de atención visual hacia estas etiquetas aumentará a medida que la bebida contenga una mayor cantidad de alcohol, especialmente si se utiliza un semáforo nutricional para mostrar el volumen de alcohol.

\section{Métodos}

\section{Diseño}

Esta investigación fue realizada mediante una serie de experimentos en laboratorio. A través de la monitorización de los movimientos oculares de los participantes se midió los niveles de atención visual hacia las diferentes etiquetas del volumen de alcohol de las cuales se manipularon su tamaño (pequeño vs. grande) y su diseño (texto vs. semáforo nutricional) como factores inter-sujetos y la graduación alcohólica $(0,4 \%, 4,6 \%$ vs. $15 \%)$ como factor intra-sujeto. Para medir la atención visual hacia la advertencia sanitaria, una submuestra, compuesta por la mitad de los participantes, también visualizó una advertencia sanitaria en los envases de las bebidas alcohólicas de la que se manipuló su tamaño (pequeño vs. grande) como factor inter-sujeto y la graduación alcohólica de la bebida $(0,4 \%, 4,6 \%$ vs. $15 \%)$ como factor intra-sujeto.

\section{Materiales y mediciones}

Estímulos. Cuatro tipos de estímulos fueron creados para mostrar las etiquetas del volumen de alcohol como combinación de los distintos factores inter-sujetos (diseño y tamaño): 1) texto, pequeño; 2) texto, grande; 3) semáforo nutricional, pequeño y 4) semáforo nutricional, grande. Posteriormente fueron incluidos en los envases de botellas de cerveza. Para los estímulos con solo texto, la información sobre el volumen de alcohol se presentó como: 'ALC. 0,4\% VOL.', 'ALC. 4,6\% VOL.' o 'ALC. $15 \%$ VOL'. Para los estímulos mostrados mediante semáforo nutricional, se usaron tres colores diferentes además del texto: verde para $0,4 \%$, amarillo para $4,6 \%$ y rojo para $15 \%$, también se incluyeron las palabras «bajo», «medio» y «alto», respectivamente. Para la mitad de los participantes, los envases de cerveza también contenían una advertencia sanitaria con el texto: «El alcohol daña tu cuerpo y tu mente». Se crearon dos tipos de advertencias sanitarias manipulando su tamaño: 1) pequeña y 2) grande. Todas estas etiquetas fueron insertadas en tres botellas de cerveza diferentes que, a su vez, se emplazaron en tres anuncios publicitarios de revista sobre las cervezas. Por tanto, se diseñaron un total de 24 botellas de cerveza completamente originales para mostrar las diferentes combinaciones de los estímulos. La Figura 1 muestras algunos ejemplos de los estímulos. El Material Complementario incluye más ejemplos de los estímulos. 


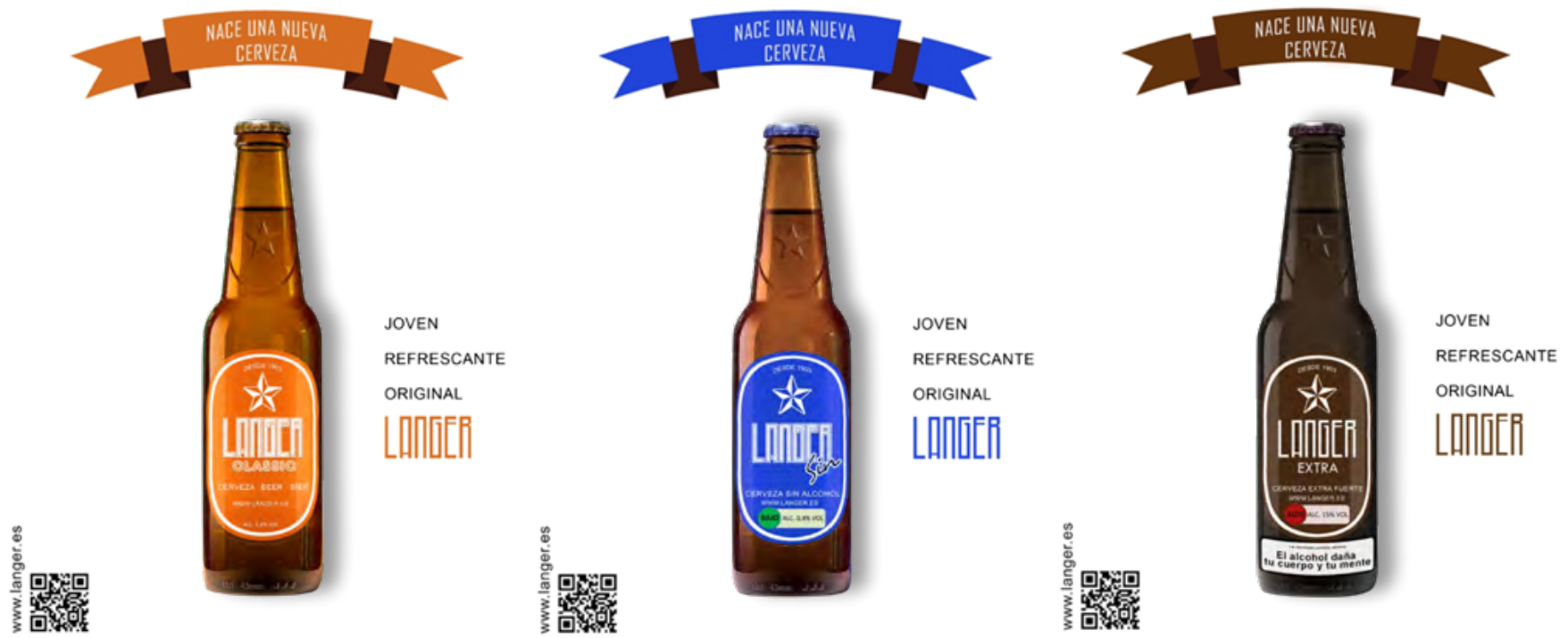

Figura 1. Ejemplos de los estímulos. A la izquierda, una botella original de cerveza de $4,6 \%$ con una etiqueta de alcohol por volumen pequeña de solo texto. En el centro, una botella original de cerveza de $0,4 \%$ con una etiqueta de alcohol por volumen grande y con semáforo nutricional. A la derecha, una botella original de cerveza de $15 \%$ con una etiqueta de alcohol por volumen grande y con semáforo nutricional; además, una advertencia sanitaria grande.

Atención visual. Se monitorizaron los movimientos oculares de los participantes mediante el equipo Tobii T120, un dispositivo de seguimiento ocular con una pantalla de 17" y frecuencia de $120 \mathrm{~Hz}$. Los movimientos oculares se midieron mediante dos cámaras binoculares de rayos infrarrojos ubicadas debajo de la pantalla del ordenador. Mediante software Tobii Studio se extrajeron el número de fijaciones hacia las etiquetas.

Otras medidas. Para fines descriptivos, los participantes también completaron el Cuestionario de Identificación de los Trastornos debidos al Consumo de Alcohol (AUDIT) (Saunders, Aasland, Babor, Fuente y Grant, 1993). Usando este cuestionario con 10 ítems, se evaluó el consumo de alcohol, el patrón de consumo y los problemas relacionados con el consumo de alcohol de los participantes ( $\alpha$ Cronbach $=0,63)$.

\section{Procedimiento}

Los participantes debían de tener una edad comprendida entre los 18-30 años y ser hispanohablantes nativos. Fueron reclutados entre los estudiantes y los empleados de la Universidad de Granada (España) y voluntarios de la población en general. Los participantes potencialmente aptos fueron invitados a participar en el estudio. En el momento del experimento, después de leer una hoja informativa, los participantes firmaron su consentimiento y completaron una serie de preguntas demográficas para conocer su edad, sexo, estado civil y nivel de estudios. Mediante una lista previamente aleatorizada, los participantes fueron asignados a uno de los cuatro diferentes grupos experimentales (para determinar el tamaño y el diseño de las etiquetas que posteriormente visualizarían) y si formaban parte de la submues- tra o del grupo de control para el estudio de la advertencia sanitaria. Posteriormente, los participantes completaron el experimento de eye-tracking precedido por una calibración de nueve puntos. El experimento de eye-tracking consistía en que los participantes tenían que visualizar una copia electrónica de la revista "Los Rolling Stones". Un total de 14 páginas fueron mostradas de forma individual en una pantalla de 17" TFT con 120x1024 pixeles. Tres de estas 14 páginas eran en los tres anuncios donde las cervezas fueron insertadas respectivamente según su graduación alcohólica $(0,4 \%, 4,6 \%$, y $15 \%)$. Estas tres páginas fueron mostradas de forma aleatoria durante el experimento. Para asegurar que los participantes se implicaban en el experimento de eye-tracking, se les pidió previamente que presentaran la mayor atención posible al contenido de la revista; ya que, al finalizar, participarían en una actividad de recuerdo. Después de terminar el experimento de eye-tracking, los participantes tomaron parte en la actividad de recuerdo similar al ejercicio de masked-recall de Thomsen y Fulton (2007) y completaron el cuestionario AUDIT.

\section{Análisis estadístico}

Para las etiquetas del volumen de alcohol, se llevó a cabo un modelo mixto ANOVA de 2 (tamaño: pequeño vs. grande) x 2 (diseño: texto vs. semáforo nutricional) $\mathrm{x}$ 3 (graduación alcohólica: 0,4\%, 4,6\% vs. 15\%) para el número de fijaciones hacia las etiquetas. Para la advertencia sanitaria, se realizó un modelo mixto ANOVA de 2 (tamaño: pequeño vs. grande) x 3 (graduación alcohólica: 0,4\%, $4,6 \%$ vs. $15 \%$ ) para el número de fijaciones hacia la advertencia. Los datos de la actividad de recuerdo no fueron analizados al no formar parte del diseño del estudio. 


\section{Resultados}

\section{Participantes}

Un total de 64 participantes $(52 \%$ mujeres; edad $=21$; $100 \%$ solteros; $61 \%$ con estudios secundarios) completaron el experimento. Los participantes obtuvieron una puntuación media en el cuestionario AUDIT de 11,17 (DT = $3,48)$, lo que indica un consumo de alcohol por encima de los límites de un consumo de bajo riesgo (Anderson, Cremona, Paton, Turner y Wallace, 1993; Babor, Higgins-Biddle, Saunders y Monteiro, 2001). La submuestra para evaluar la advertencia sanitaria estuvo compuesta por 32 participantes.

\section{Atención visual}

En comparación con las etiquetas del volumen de alcohol más pequeñas, las etiquetas más grandes obtuvieron mayores niveles de atención visual $\left(\mathrm{F}_{(1,64)}=41,99, \eta \mathrm{p}^{2}=\right.$ 0,41, $\mathrm{p}<, 001)$. Aunado a ello, también se halló un aumento en el número de fijaciones mediante el uso del semáforo nutricional $\left(\mathrm{F}_{(1,64)}=10,50, \eta \mathrm{p}^{2}=0,41, \mathrm{p}=, 002\right)$ en comparación con el diseño de solo texto. Se halló una evidencia estadística suficiente para la interacción entre tamaño y diseño $\left(\mathrm{F}_{(1,64)}=4,67, \eta \mathrm{p}^{2}=0,072, \mathrm{p}=, 035\right)$, de tal manera que la atención visual aumentó cuando el etiquetado del volumen de alcohol se mostraba con un mayor tamaño y mediante un semáforo nutricional. Por último, no se encontró una evidencia estadística clara para una diferencia en el número de fijaciones diferente según los distintos niveles de graduación alcohólica $\left(\mathrm{F}_{(2,64)}=1,81, \eta \mathrm{p}^{2}=0,03\right.$, $\mathrm{p}=, 17)$ o para el efecto interacción entre la graduación alcohólica y el diseño $\left(\mathrm{F}_{(2,64)}=0,85, \eta \mathrm{p}^{2}=0,08, \mathrm{p}=, 54\right)$. La Tabla 1 muestra los resultados.

Con una evidencia estadística clara, se observó que el tamaño de la advertencia sanitaria tuvo un efecto positivo sobre el número de fijaciones $\left(\mathrm{F}_{(1,32)}=15,18, \eta \mathrm{p}^{2}=, 34, \mathrm{p}\right.$ $<, 001)$. También se halló un efecto de la graduación alcohólica sobre el nivel de atención visual $\left(\mathrm{F}_{(2,32)}=7,52, \eta \mathrm{p}^{2}=\right.$ $0,20, p=, 001)$ : las advertencias ubicadas en los envases con una graduación alcohólica de $4,6 \%$ obtuvieron el mayor número de fijaciones, seguido por el envases con $15 \%$, y finalmente los envases con una graduación del 0,4\%.

\section{Discusión}

Desde el conocimiento de los investigadores, esta es la primera investigación que examina cómo mejorar el etiquetado del alcohol en España, y uno de los pocos a nivel mundial que usa para ello medidas objetivas como es el seguimiento ocular. Como sugieren tanto este estudio como la literatura publicada previamente, el etiquetado de alcohol en Europa, que con frecuencia es implementado por la industria del alcohol de manera voluntaria, es insuficiente. Nuestro estudio muestra que, en España, como en la mayoría de los países europeos donde las etiquetas del volumen de alcohol son pequeñas y con solo texto, estas etiquetas atraen bajos niveles de atención. Incrementando el tamaño y usando un semáforo nutricional, las etiquetas del volumen de alcohol serían capaces de atraer mayores niveles de atención. Investigaciones previas apoyan estos resultados (Blackwell et al., 2018; Farke, 2011; Graham et al., 2012; Kersbergen y Field, 2017). En España, como en la mayoría de los países europeos, las bebidas alcohólicas no están obligadas a incluir advertencias sanitarias acerca de los riesgos del consumo de alcohol (Farke, 2011), a pesar de su potencial para comunicar los perjuicios asociados al consumo de alcohol y para disuadir patrones de consumo de riesgo entre los consumidores (Blackwell et al., 2018). En cuanto a la posibilidad de incluir este tipo de adverten-

Tabla 1. Número de fijaciones

\begin{tabular}{|c|c|c|c|c|c|c|}
\hline \multirow{2}{*}{\multicolumn{2}{|c|}{ Etiqueta de alcohol por volumen }} & \multirow{2}{*}{$\begin{array}{c}\text { Total } \\
(\mathrm{n}=64)\end{array}$} & \multicolumn{2}{|c|}{ Tamaño } & \multicolumn{2}{|c|}{ Diseño } \\
\hline & & & $\begin{array}{l}\text { Pequeño } \\
(\mathrm{n}=32)\end{array}$ & $\begin{array}{l}\text { Grande } \\
(\mathrm{n}=32)\end{array}$ & $\begin{array}{c}\text { Texto } \\
(\mathrm{n}=32)\end{array}$ & $\begin{array}{c}\text { Semáforo } \\
(\mathrm{n}=32)\end{array}$ \\
\hline \multirow{4}{*}{ Graduación alcohólica } & $0,4 \%$ & $0,97(1,48)$ & $0,38(0,61)$ & $1,56(1,83)$ & $0,47(0,72)$ & $1,47(1,85)$ \\
\hline & $4,6 \%$ & $0,81(1,18)$ & $0,34(0,55)$ & $1,28(1,44)$ & $0,50(0,80)$ & $1,06(1,44)$ \\
\hline & $15 \%$ & $1,16(1,39)$ & $0,34(0,60)$ & $1,97(1,49)$ & $0,97(1,40)$ & $1,34(1,38)$ \\
\hline & Total & $0,98(1,32)$ & $0,35(0,59)$ & $1,60(1,59)$ & $0,67(, 97)$ & $1,29(1,56)$ \\
\hline \multirow{2}{*}{\multicolumn{2}{|c|}{ Etiqueta de advertencia sanitaria }} & & \multicolumn{2}{|c|}{ Tamaño } & & \\
\hline & & $\begin{array}{l}\text { Total } \\
(\mathrm{n}=32)\end{array}$ & $\begin{array}{l}\text { Pequeño } \\
(n=16)\end{array}$ & $\begin{array}{l}\text { Grande } \\
(n=16)\end{array}$ & & \\
\hline \multirow{4}{*}{ Graduación alcohólica } & $0,4 \%$ & $1,81(1,75)$ & $1,38(1,50)$ & $2,25(1,91)$ & & \\
\hline & $4,6 \%$ & $3,47(3,34)$ & $1,63(1,93)$ & $5,31(3,48)$ & & \\
\hline & $15 \%$ & $2,41(2,56)$ & $1,00(1,26)$ & $3,81(2,79)$ & & \\
\hline & Total & $2,56(2,55)$ & $1,33(1,56)$ & $3,79(2,72)$ & & \\
\hline
\end{tabular}

Nota. Número medio de fijaciones a la etiqueta del volumen de alcohol según tamaño (pequeño vs. grande), diseño (solo texto vs. semáforo nutricional) y graduación alcohólica (0,4\%, 4,6\% y 15\%), y número medio de fijaciones a la advertencia sanitaria según tamaño (pequeño vs. grande) y graduación alcohólica (o,4\%, 4,6\% y $15 \%)$. Las desviaciones típicas aparecen entre paréntesis. 
cias sanitarias en los envases de alcohol, los datos de esta investigación sugieren que las advertencias deberían de ser mostradas lo suficientemente grandes como para poder atraer la atención de los consumidores.

Estudios previos han demostrado una clara influencia de los factores relacionados con las características básicas del estímulo (factores bottom-up) en la atención, principalmente de forma involuntaria (Wedel y Pieters, 2008). Esto explicaría en gran medida los resultados obtenidos: al aumentar el tamaño de los estímulos y al incluir el diseño de un semáforo nutricional se cambiaron las características básicas de los estímulos lo que, como se esperaba, produjo un aumento en la atención prestada a los mismos. No obstante, esto no explicaría por qué la advertencia sanitaria obtuvo los mayores niveles de atención visual en los envases con una graduación alcohólica de 4,6\%. Es probable que otros factores, relacionados con el individuo (factores top-down), también jueguen un papel importante. Por ejemplo, investigaciones previas sobre tabaco hallaron que los fumadores evitan de manera activa prestar atención a las advertencias sanitarias en los envases (Maynard et al., 2014). Una mayor investigación es necesaria en esta dirección.

Como un estudio preliminar que explora el impacto del etiquetado del alcohol en la atención visual, existen ciertas limitaciones. Primero, esta investigación fue realizado en España, donde los sistemas de unidades de bebida estándar para mostrar el contenido de alcohol no se aplican. De tal manera que no se pudo examinar el impacto de dichos sistemas sobre la atención visual. Segundo, solo se evaluó la inclusión de un solo tipo de advertencia sanitaria en una submuestra del estudio; por tanto, los resultados relacionados con este estímulo han de ser interpretados con cautela. Investigaciones futuras deben explorar un mayor rango de advertencias diferentes, incluyendo el riesgo de consumir alcohol durante el embarazo y otros tipos de mensajes. También hay limitaciones inherentes debido a la edad de los participantes y al tipo de bebida alcohólica usada. Estas pueden limitar extrapolar los resultados a otras poblaciones u otras bebidas alcohólicas. Por último, aunque la atención es el primer paso en el procesamiento de las etiquetas, estudios futuros deberían examinar como la atención visual se relaciona con la conducta final del consumo de alcohol.

A pesar de estas limitaciones, existen importantes implicaciones. Como el primer estudio que examina el etiquetado de las bebidas alcohólicas en España, los resultados sugieren que las etiquetas actuales (pequeñas y con solo texto) pasan relativamente desapercibidas por los consumidores. Investigaciones previas han mostrado que el entorno urbano de Cataluña promueve el consumo de alcohol al estar caracterizado por su alta disponibilidad, por lo que serían necesario mejoras en la legislación que pudieran permitir cambiar su imagen social (Villalbi et al., 2019). Esto es particularmente importante dado los fracasos por conseguir acuerdos volun- tarios con la industria del alcohol y la recomendación de mayor legislación sobre el etiquetado de bebidas alcohólicas (European Union Committee, 2015). En este contexto, recientemente en España se han llevado a cabo importantes esfuerzos para desarrollar una nueva regulación sobre el etiquetado del alcohol (Congreso de los Diputados, 2018). Este estudio ofrece una serie de pautas para que una futura regulación sobre el etiquetado del alcohol garantice que el diseño de las etiquetas sea capaz de atraer la atención visual, incrementando su tamaño y mediante el uso de diseños gráficos como un semáforo nutricional.

\section{Conflicto de intereses}

Los autores declaran la inexistencia de conflictos de intereses.

\section{Referencias}

Anderson, P., Cremona, A., Paton, A., Turner, C. y Wallace, P. (1993). The risk of alcohol. Addiction, 88, 1493-1508. doi:10.1111/j.1360-0443.1993.tb03135.x.

Anderson, P., Moller, L., Galea, G. y World Health Organization (Eds.). (2012). Alcohol in the European Union: consumption, harm and policy approaches. Copenhagen, Denmark: World Health Organization Regional Office for Europe.

Babor, T. F., Higgins-Biddle, J. C., Saunders, J. B. y Monteiro, M. G. (2001). The Alcohol Use Disorders Identification Test: Guidelines for use in. 2nd ed. World Health Organization. Recuperado de https://apps.who.int/iris/ handle/10665/67205.

Blackwell, A. K. M., Drax, K., Attwood, A. S., Munafo, M.R. y Maynard, O. M. (2018). Informing drinkers: Can current UK alcohol labels be improved? Drug and Alcohol Dependence, 192, 163-170. doi:10.1016/j. drugalcdep.2018.07.032.

Congreso de los Diputados. (2018, April 24). Comision Mixta para el Estudio del Problema de las Drogas Sesion no 10 24/04/2018. Recuperado de http://www. congreso.es/wc/wc/audiovisualdetalledisponible?codS esion=10\&-codOrgano=322\&fechaSesion $=24 / 04 / 2018$ \&mp4=mp4\&idLegislaturaElegida=12.

European Union Committee. (2015). A New EU Alcohol Strategy? Recuperadodehttps:/ / publications.parliament. uk/pa/ld201415/ldselect/ldeucom/123/12302.htm.

Farke, W. (2011). Health warnings and responsibility messages on alcoholic beverages-a review of practices in Europe. Mainz Germany: German Catholic University of Applied Sciences.

Graham, D. J., Orquin, J. L. y Visschers, V. H. M. (2012). Eye tracking and nutrition label use: A review of the literature and recommendations for label enhancement. Food Policy, 37, 378-382. doi:10.1016/j.foodpol.2012.03.004. 
Hammond, D. (2011). Health warning messages on tobacco products: a review. Tobacco Control, 20, 327-337. doi:10.1136/tc.2010.037630.

Kersbergen, I. y Field, M. (2017). Alcohol consumers' attention to warning labels and brand information on alcohol packaging: Findings from cross-sectional and experimental studies. BMC Public Health, 17. doi:10.1186/s12889-017-4055-8.

Maynard, O. M., Attwood, A., O’Brien, L., Brooks, S., Hedge, C., Leonards, U. y Munafo, M. R. (2014). Avoidance of cigarette pack health warnings among regular cigarette smokers. Drug and Alcohol Dependence, 136, 170-174. doi:10.1016/j.drugalcdep.2014.01.001.

Noar, S. M., Hall, M. G., Francis, D. B., Ribisl, K. M., Pepper, J. K. y Brewer, N. T. (2015). Pictorial cigarette pack warnings: a meta-analysis of experimental studies. Tobacco Control, 25, 341-354. doi:10.1136/ tobaccocontrol-2014-051978.

Parlamento Europeo y del Consejo. Reglamento (UE) $\mathrm{n}^{\circ}$ 1169/2011 del Parlamento Europeo y del Consejo de 25 de octubre de 2011 sobre la informacion alimentaria facilitada al consumidor y por el que se modifican los Reglamentos (CE) n ${ }^{\circ}$ 1924/2006 y (CE) n ${ }^{\circ}$ 1925/2006 del Parlamento Europeo y del Consejo, y por el que se derogan la Directiva 87/250/CEE de la Comision, la Directiva 90/496/CEE del Consejo, la Directiva 1999/10/CE de la Comisión, la Directiva 2000/13/CE del Parlamento Europeo y del Consejo, las Directivas 2002/67/CE, y 2008/5/CE de la Comision, y el Reglamento (CE) $n^{\circ}$ 608/2004 de la Comision, Pub. L. $\mathrm{n}^{\circ}$ 1169, 46 (2011). Recuperado de https://www.boe. es/doue/2011/304/L00018-00063.pdf.

Saunders, J. B., Aasland, O. G., Babor, T. F., Fuente, J. R. D. L. y Grant, M. (1993). Development of the Alcohol Use Disorders Identification Test (AUDIT): WHO Collaborative Project on Early Detection of Persons with Harmful Alcohol Consumption - II. Addiction, 88, 791804. doi:10.1111/j.1360-0443.1993.tb02093.x.

Thomsen, S. R. y Fulton, K. (2007). Adolescents' Attention to Responsibility Messages in Magazine Alcohol Advertisements: An Eye-Tracking Approach. Journal of Adolescent Health, 41, 27-34. doi:10.1016/j. jadohealth.2007.02.014.

Thomson, L. M., Vandenberg, B. y Fitzgerald, J. L. (2012). An exploratory study of drinkers views of health information and warning labels on alcohol containers: Warning labels on alcohol containers. Drug and Alcohol Review, 31, 240-247. doi:10.1111/j.14653362.2011.00343.x.

Truitt, L., Hamilton, W. L., Johnston, P. R., Bacani, C. P., Crawford, S. O., Hozik, L. y Celebucki, C. (2002). Recall of health warnings in smokeless tobacco ads. Tobacco Control, 11, ii59-ii63. doi:10.1136/tc.11.suppl_2.ii59.
Villalbi, J. R., Espelt, A., Sureda, X., Bosque-Prous, M., Teixido-Compano, E., Puigcorbe, S., ... Brugal, M. T. (2019). The urban environment of alcohol: a study on the availability, promotion and visibility of its use in the neighborhoods of Barcelona. Adicciones, 31, 33-40. doi:10.20882/adicciones.950.

Wedel, M. y Pieters, R. (2008). A review of eye-tracking research in marketing. En Review of marketing research (pp. 123-147). Emerald Group Publishing Limited.

Wilkinson, C., Allsop, S., Cail, D., Chikritzhs, T., Daube, M., Kirby, G. y Mattick, R. (2009). Alcohol Warning Labels: Evidence of impact on alcohol consumption amongst women of childbearing age. Food Standards Australia New Zealand, Canberra.

Wilkinson, C. y Room, R. (2009). Warnings on alcoholcontainers and advertisements: international experience and evidence on effects. Drug and Alcohol Review, 28, 426435. doi:10.1111/j.1465-3362.2009.00055.x. 